\title{
Planar Cell Polarity Protein Vangl2 Interacts With Protein Ap2m1 to Regulate Dendritic Branching in Cortical Neurons
}

\author{
Misato Yasumura \\ Osaka University
}

Akari Hagiwara

University of Yamanashi

Yamato Hida

University of Yamanashi

Toshihisa Ohtsuka ( $\nabla$ tohtsuka@yamanashi.ac.jp)

University of Yamanashi

\section{Research Article}

Keywords: PCP, Van Gogh-like proteins, adaptor-related protein complex, yeast two-hybrid

Posted Date: January 18th, 2021

DOI: https://doi.org/10.21203/rs.3.rs-145755/v1

License: (c) (i) This work is licensed under a Creative Commons Attribution 4.0 International License. Read Full License 


\section{Abstract}

Van Gogh-like 2 (Vangl2) is a mammalian homolog of Drosophila core planar cell polarity (PCP) protein Vang/Strabismus, which organizes asymmetric cell axes for developmental proliferation, fate determination, and polarized movements in multiple tissues, including neurons. While the PCP pathway has an essential role for dendrite and dendritic spine formation, the molecular mechanism remains to be clarified. To investigate the mechanism of Vangl2-related neuronal development, we screened for proteins that interact with the Vangl2 cytosolic N-terminus from postnatal day 9 mouse brains using a yeast twohybrid system. From 61 genes, we identified adaptor-related protein complex 2, mu 1 subunit (Ap2m1) as the Vangl2 N-terminal binding protein. Intriguingly, however, the pull-down assay demonstrated that Vangl2 interacted with Ap2m1 not only at its $\mathrm{N}$-terminus but also at the $\mathrm{C}$-terminal Prickle binding domain. Furthermore, we verified that the downregulation of Ap2m1 in the developing cortical neurons reduced the dendritic branching similar to what occurs in a knockdown of Vangl2. From these results, we suggest that the membrane internalization regulated by the PCP pathway is required for the developmental morphological change in neurons.

\section{Introduction}

Van Gogh (Vang), also known as Strabismus (Stbm), was originally identified in Drosophila as a core planar cell polarity (PCP) protein, which when mutated, causes considerable misorientation of organized epithelial structures (Torban et al., 2004; Tissir \& Goffinet, 2013). Vang/Stbm is evolutionarily conserved, and can be seen in several species including insects, fish, and mammals. Mice have two Vang/Stbm family members: Van Gogh-like protein (Vangl) 1 and Vangl2, whose mRNAs are expressed in the developing and adult nervous system (Tissir \& Goffinet, 2006). Mutations in VANGL genes have been identified in sporadic and familial cases of neural tube defects in humans, and similar defects have been found in the looptail $(L P)$ mouse mutant that has mutations in Vangl2.

Vangl2 is a membrane protein comprising four transmembrane domains and two intracellular domains, one each at the amino (N)- and carboxyl (C)-terminals. The $\mathrm{C}$-terminus has multiple domains including the PDZ-binding motif, the Prickle binding domain, and some missense mutations that cause the $L p$ mutant (Torban et al., 2004). Indeed, two independent $L p$ mutations in Vangl2 have been shown to impair interactions with Dvl proteins, which suggests that Vangl2-Dvl-mediated signaling underlies the neural tube defect (Torban et al., 2004). In the nervous system, Vangl2 regulates commissural axon growth-cone guidance by antagonizing Dvl-mediated signaling (Shafer et al., 2011). Additionally, the Vangl2 PDZbinding motif is tightly associated with postsynaptic density (PSD)-95 protein. They form a protein complex with NMDA receptors, Prickle2, and N-cadherin, which regulate the clustering of postsynaptic molecules and dendritic spine formation (Yoshioka et al., 2013; Nagaoka, Ohashi, et al., 2014; Nagaoka et al., 2015; Nagaoka \& Kishi, 2016). Intriguingly, the Vangl2 C-terminal deletion mutant was shown to enhance dendritic branching, while the $\mathrm{N}$-terminus deletion mutant reduced both spine density and dendritic branching (Hagiwara et al., 2014), which indicates that Vangl2 has an essential role in modulating the constitution of neuronal morphology. 
Vangl2 plays critical roles in early neural development and axon/dendritic branching. However, despite these broad-ranging functions, the functional domains and proteins that it interacts with (other than PCP proteins) have not been well studied. Unlike the well-characterized domains of the C-terminal region, which include a region that interacts with PCP partner proteins (Bailly et al., 2018), the roles of the Nterminal region remain poorly characterized. Here, we identified proteins that interact with the N-terminus of Vangl2 (Vangl2N) using yeast two-hybrid screening. Following the pull-down assay, we found proteins that interact with Vangl2: adaptor-related protein complex 2, mu1 subunit (Ap2m1), eukaryotic translation elongation factor 1 a1 (Eef1a1), and Ras/Rap GTPase-activating protein SynGAP (SynGAP1). Ap2m1 was found to be the most likely to interact with Vangl2N, binding to both the N-terminus and the Cterminal Prickle binding domain. Remarkably, knockdown (KD) of Ap2m1 resulting in less branching of cortical neurons, similar to what happens in the KD of Vangl2. Because the AP2 protein regulates membrane internalization via clathrin-mediated endocytosis, the Vangl2-involved PCP signal coordinates the temporal and spatial regulation of neuronal morphology.

\section{Results}

\section{Screening of novel proteins that interact with Vangl2}

To search for novel Vangl2 binding proteins, we conducted yeast two-hybrid screening with the N-terminal region (amino acid 1-114, Vangl2N) as bait, and mated it with a postnatal day 9 (P9) mouse brain cDNA library containing the $\mathrm{Y} 187$ yeast strain (Fig. 1A, B). The resulting diploid cells were screened on different stringency plates, and approximately $4.5 \times 10^{7}$ diploid cells were cultured. Among 213 positively identified clones, we identified 61 genes by sequencing and BLAST searches (Fig. 1B, Table 1). To investigate how the $\mathrm{N}$-terminal region of Vangl2 regulates dendrites and/or dendritic spines formation, we selected 10 proteins from those that were identified, choosing them based on proteomic data from postsynaptic density (PSD) proteins in the P9 mouse cortex (Shao et al., 2017) (Table 1 indicated by *). Among them, two housekeeping proteins, one nuclear protein, and two presynaptic proteins were excluded from further analysis. As for the remaining five proteins, Ap2m1, ATPase $\mathrm{Na}^{+} / \mathrm{K}^{+}$transporting subunit $\beta 1$ (Atp1b1), Eef1a1, kinesin family member 1A (Kif1a), and SynGAP1, we examined how they interacted with the N-terminal region of Vangl2. We performed pull-down assays using purified GSTVangI2N and GST-Vangl2C (amino acid 242-521) with cell lysates from HEK293 cells that expressed amino-terminally FLAG-tagged protein. Cell lysates expressing FLAG-Ap2m1 or FLAG-SynGAP1 were mostly retained on GST-Vangl2N, with fewer retained on GST-Valng2C. Conversely, cell lysates expressing Eef1a1 interacted with both GST-Vangl2N and GST-Vangl2C, while those expressing Atp1b1 and Kif1a were retained the least (Fig. 1C, Supplementary Fig. 1, Table 2). Considering the amount of pulled down FLAG-tagged protein, we focused on Ap2m1 for further analysis. 
Gene symbol

Name

Synaptic localization (pre/post-synaptic)

(1) Receptors, channels, and transporters

$1 \quad$ Astn1

2 Atp $1 \mathrm{~b} 1^{*}$

$3 \quad$ Atp1b2

$4 \quad$ Gria1

$5 \quad$ Grip2 astritactin 1

ATPase, $\mathrm{Na} / \mathrm{K}$ transporting, beta 1 polypeptide

ATPase, $\mathrm{Na} / \mathrm{K}$ transporting, beta 2 polypeptide

Glutamate receptor 1

glutamate receptor interacting protein 2

(2) Scaffold, adaptor, and membrane-trafficking proteins

\begin{tabular}{llll}
6 & Ap2m1 $^{*}$ & $\begin{array}{l}\text { adaptor-related protein complex 2, mu } 1 \\
\text { subunit }\end{array}$ & pre/post \\
\hline 7 & Pclo* $^{*}$ & protein piccolo & pre \\
\hline 8 & Syt2 & synaptotagmin-2 & pre \\
9 & Syt4 & synaptotagmin-4 & pre/post \\
10 & Syt7 & synaptotagmin-7 & pre \\
11 & Syt11 & synaptotagmin-11 & post
\end{tabular}

(3) Kinases/phosphatases and regulators

12 Ctdnep1 CTD nuclear envelope phosphatase 1

13 Ptprm receptor-type tyrosine-protein

phosphatase mu

(4) Small G-proteins, GTPase, ATPase, and regulators
14 Syngap1 ${ }^{*}$
Ras/Rap GTPase-activating protein
SynGAP
post

(5) Motor proteins

$\begin{array}{llll}15 & \text { Clstn1 } & \text { calsyntenin } 1 & \text { pre/post } \\ 16 & \text { Kif1a } & \text { kinesin family member 1A } & \text { pre/post }\end{array}$

(6) Cell adhesion proteins

*Proteins identified from the postsynaptic density of the P9 mouse cortex. 


\begin{tabular}{|c|c|c|c|}
\hline & Gene symbol & Name & $\begin{array}{l}\text { Synaptic localization } \\
\text { (pre/post-synaptic) }\end{array}$ \\
\hline 17 & Cadm3 & cell adhesion molecule 3 , syncam 3 & pre \\
\hline 18 & Ctnnd1 & catenin, delta 1 & pre/post \\
\hline 19 & Itga6 & integrin alpha 6 & \\
\hline 20 & Ncam2 & neural cell adhesion molecule 2 & \\
\hline 21 & Pcdh1 & protocadherin 1 & \\
\hline 22 & Pcdha2 & protocadherin alpha 2 & \\
\hline 23 & Pcdhgc3 & protocadherin gamma subfamily $\mathrm{C}, 3$ & \\
\hline 24 & Pecam1 & $\begin{array}{l}\text { platelet endothelial cell adhesion } \\
\text { molecule }\end{array}$ & \\
\hline 25 & Pvrl2 & nectin cell adhesion molecule 2 & \\
\hline 26 & Pvrl3 & nectin cell adhesion molecule 3 & \\
\hline \multicolumn{4}{|c|}{ (7) Chaperone/folding proteins } \\
\hline 27 & Dnaja1 & DnaJ Hsp40 member A1 & \\
\hline 28 & Fkbp1b & FK506 binding protein 1b & \\
\hline \multicolumn{4}{|c|}{ (8) Metabolism-related proteins } \\
\hline 29 & B4galnt1 & $\begin{array}{l}\text { beta-1,4-N-acetyl-galactosaminyl } \\
\text { transferase } 1\end{array}$ & \\
\hline 30 & Cds1 & CDP-diacylglycerol synthase 1 & \\
\hline 31 & Gapdh $^{*}$ & $\begin{array}{l}\text { Glyceraldehyde-3-phosphatase } \\
\text { dehydrogenase }\end{array}$ & \\
\hline 32 & Masp1 & mannan-binding lectin serine peptidase 1 & \\
\hline 33 & Pomgnt1 & $\begin{array}{l}\text { protein O-linked mannose beta-1,2-N- } \\
\text { acetylglucosaminyltransferase } 1\end{array}$ & \\
\hline 34 & Ube2m & NEDD8-conjugating enzyme Ubc 12 & \\
\hline \multicolumn{4}{|c|}{ (9) Mitochondrial proteins } \\
\hline 35 & Timm23 & $\begin{array}{l}\text { mitochondrial import inner membrane } \\
\text { translocase subunit Tim23 }\end{array}$ & \\
\hline
\end{tabular}

*Proteins identified from the postsynaptic density of the P9 mouse cortex. 


\begin{tabular}{|c|c|c|c|}
\hline & Gene symbol & Name & $\begin{array}{l}\text { Synaptic localization } \\
\text { (pre/post-synaptic) }\end{array}$ \\
\hline 36 & Eef1a $1^{*}$ & $\begin{array}{l}\text { eukaryotic translation elongation factor } \\
\text { alpha } 1\end{array}$ & post \\
\hline 37 & Gltf3c5 & $\begin{array}{l}\text { general transcription factor IIIC, } \\
\text { polypeptide } 5\end{array}$ & \\
\hline 38 & KIf13 & kruppel-like factor 13 & \\
\hline 39 & Mkl2 & myocardin related transcription factor $B$ & \\
\hline 40 & Pcbp2* & poly $(\mathrm{rC})$ binding protein 2 & \\
\hline 41 & Scaf8 & SR-related and CTD-associated factor 8 & \\
\hline 42 & Zmiz1 & $\begin{array}{l}\text { zinc finger MIZ domain-containing protein } \\
1\end{array}$ & \\
\hline 43 & Zmiz2 & $\begin{array}{l}\text { zinc finger MIZ domain-containing protein } \\
\end{array}$ & \\
\hline 44 & 2700060E02Rik(Rtraf) & RNA transcription, translation and transport & factor \\
\hline \multicolumn{4}{|c|}{ (11) DNA/nucleus/histone proteins } \\
\hline 45 & Hnmph3 & $\begin{array}{l}\text { heterogeneous nuclear ribonucleoprotein } \\
\text { H3 }\end{array}$ & \\
\hline 46 & Hnmpul1 & $\begin{array}{l}\text { heterogeneous nuclear ribonucleoprotein } \\
\text { U-like } 1\end{array}$ & \\
\hline 47 & $H 1 f x$ & $\mathrm{H} 1.10$ linker histone & \\
\hline 48 & Nrbp1 & nuclear receptor binding protein 1 & \\
\hline 49 & Papola & poly A polymerase alpha & \\
\hline 50 & Papolb & poly A polymerase beta & \\
\hline \multicolumn{4}{|c|}{ (12) Others } \\
\hline 51 & Anapc10 & anaphase promoting complex subunit 10 & \\
\hline 52 & Atg2b & autophagy related $2 \mathrm{~b}$ & \\
\hline 53 & Efr3b & EFR homolog B & post \\
\hline 54 & Hdlbp & high density lipoprotein binding protein & \\
\hline 55 & Kpna6 & karyopherin alpha 6 & \\
\hline 56 & Nrep & neuronal regeneration related protein & \\
\hline
\end{tabular}

*Proteins identified from the postsynaptic density of the P9 mouse cortex. 


\begin{tabular}{|c|c|c|c|}
\hline & Gene symbol & Name & $\begin{array}{l}\text { Synaptic localization } \\
\text { (pre/post-synaptic) }\end{array}$ \\
\hline 57 & Tmem98 & transmembrane protein 98 & \\
\hline 58 & Tmem132a & transmembrane protein $132 a$ & \\
\hline 59 & Ywhaz ${ }^{*}$ & 14-3-3 protein zeta/delta & \\
\hline 60 & Zfyve9 & $\begin{array}{l}\text { zinc finger FYVE domain-containing } \\
\text { protein }\end{array}$ & \\
\hline 61 & 2510009E07Rik & & \\
\hline
\end{tabular}

Table 2

Pulldown assay for the Vangl2 $\mathrm{N}$ - and C-terminal

\begin{tabular}{|c|c|c|c|c|c|}
\hline & $\begin{array}{l}\text { Gene } \\
\text { symbol }\end{array}$ & Name & $\begin{array}{l}\mathrm{MW} \\
(\mathrm{kDa})\end{array}$ & Vangl2N & Vangl2C \\
\hline 1 & Atp1b1 & ATPase $\mathrm{Na} / \mathrm{K}$ transporting 1 polypeptide & 35 & + & + \\
\hline 2 & Ap2m1 & $\begin{array}{l}\text { adaptor-related protein complex 2, mu1 } \\
\text { subunit }\end{array}$ & 50 & +++ & + \\
\hline 3 & Eef1a1 & $\begin{array}{l}\text { eukaryotic translation elongation factor } \\
\text { alpha } 1\end{array}$ & 50 & ++ & ++ \\
\hline 4 & Syngap1 & Ras/Rap GTPase-activating protein synGAP & 148 & ++ & - \\
\hline 5 & Kif1a & Kinesin family member $1 \mathrm{~A}$ & 202 & - & - \\
\hline
\end{tabular}

\section{The Region Of Vangl2 That Interacted With Ap2m1}

Yeast two-hybrid screening allowed us to isolate four independent cDNA clones encoding Ap2 1 1. These clones contained fragments of Ap2m1 that encoded the amino acids 232-367, 198-324, 224-363, and 195-367. To examine whether the fragment of Ap2m1 that encoded the amino acids from 195 to 367 was sufficient for binding to the N-terminal region of Vangl2, we performed pull-down assays using purified GST-Ap2m1 (195-367aa) with cell lysates from HEK293 cells that expressed the amino-terminally HA-tagged Vangl2 deletion mutant. Cell lysates from HEK293 cells that expressed HA-Vangl2 or Vangl2 lacking the C-terminal region (Vangl2 $\Delta \mathrm{C}$ ) were retained on GST-Ap2m1 (195-367aa) (Fig. 2A, B). The interaction of the Ap2m1 fragment with Vangl2 was also observed in HEK293 cells co-transfected with EGFP-tagged Ap2m1 fragments and HA-tagged Vangl2 (Fig. 2C). These results indicated that the region of Ap2m1 that encodes the amino acids from 195 to 367 was sufficient for the interaction with Vangl2. 
Unlike the results in Fig. 1C, Vangl2 $\mathrm{N}$ was retained on GST-Ap2m1 (195-367aa) from cell lysates expressing HA-Vangl2 $\Delta N$ (Fig. 2A, B, Supplementary Fig. 1). To identify the region of Vangl2 responsible for the interaction with Ap2m1, we performed pull-down assays with multiple regions using purified GSTVangl2 fragments and cell lysates from HEK293 cells that expressed FLAG-Ap2m1 (Fig. 3A). GST-Vangl21 and GST-Vangl2N retained FLAG-Ap2m1 from the cell lysates, but GST-Vangl2-2 did not (Fig. 3B, Supplementary Fig. 1). Similarly, GST-VangI2C and GST-Vangl2-4, but not GST-Vangl2-3, -4 , -5 , or -6 retained FLAG-Ap2m1 from the cell lysates (Fig. 3B). The amount of pulled down FLAG-Ap2m1 via GSTVangl2-1 was more than that via GST-Vangl2-4. These results suggested that Ap2m1 binds strongly to Vangl2 through the N-terminal 1-65aa region and binds weakly through the C-terminal 298-382aa region, which corresponds to the Prickle binding domain.

\section{The Functional Role Of Ap2m1 In Neuronal Dendritic Branching}

From the yeast two-hybrid screening, we found an association between Vangl2 and the adaptor-related protein complex, AP2. To evaluate the role of AP2 on the morphology of neurons, an shRNA construct was designed to downregulate endogenous Ap2m1. The efficiency of Ap2m1 shRNA was first confirmed by transfection in HEK293 cells (Fig. 4A, B, Supplementary Fig. 1). Next, we electroporated the Ap2m1 shRNA construct with EGFP into embryonic cortical neurons (E14.5-15.5). At P21, we characterized the morphology of cortical pyramidal neurons identified with EGFP. The total length of apical and basal dendrites was significantly lower in Vangl2 and Ap2m1 KD neurons than that in control with scramble shRNA (Fig. 4C, D, Table 3). Conversely, spine density was higher in Ap2m1 KD neurons compared to the comtrol and Vanlg2 KD (Fig. 4E). The total number of tips was significantly lower in Vangl2 and Ap2m1 KD neurons than control; branches at the secondary dendrite were especially devoid of tips (Fig. 4F, G). These results suggest an association between the Vangl2 portion of PCP proteins and an adaptor protein for membrane internalization that might have a role in the dendritic remodeling in neurons, which can affect neuronal development and plasticity.

Table 3 Morphological analysis of the dendritic formation 


\begin{tabular}{|llll|}
\hline \multicolumn{4}{|c|}{ Apical dendrite length $(\mu \mathrm{m})$} \\
\hline & Primary & Secondary & Tertiary \\
\hline Scramble & $16.5 \pm 1.6$ & $57.4 \pm 6.6$ & $93.5 \pm 19.1$ \\
\hline shVangl2 & $27.3 \pm 4.4$ & $45.9 \pm 17.4$ & $120.9 \pm 26.0$ \\
\hline shAp2m1 & $24.7 \pm 3.0$ & $38.0 \pm 6.6$ & $58.6 \pm 8.5$ \\
\hline Basal dendrite length $(\mu \mathrm{m})$ & \\
\hline Scramble & $21.1 \pm 1.5$ & $52.3 \pm 4.1$ & $60.8 \pm 5.5$ \\
\hline shVangl2 & $21.9 \pm 2.6$ & $41.7 \pm 5.4$ & $80.7 \pm 16.4$ \\
\hline shAp2m1 & $22.6 \pm 1.8$ & $40.7 \pm 3.8$ & $77.8 \pm 15.6$ \\
\hline
\end{tabular}

\begin{tabular}{|c|c|c|c|c|c|}
\hline \multicolumn{4}{|c|}{ Total dendrite length $(\mu \mathrm{m})$} & \multirow[t]{2}{*}{ Mouse number } & \multirow[t]{2}{*}{ Neuron numbe } \\
\hline & Apical & Basal & Total & & \\
\hline Scramble & $262.8 \pm 29.7$ & $82.7 \pm 5.5$ & $690.7 \pm 39.9$ & 4 & 17 \\
\hline shVangl2 & $183.8 \pm 23.4$ & $54.0 \pm 8.9$ & $399.6 \pm 64.7$ & 2 & 8 \\
\hline shAp2m1 & $186.9 \pm 25.1$ & $83.5 \pm 10.6$ & $508.8 \pm 54.9$ & 3 & 14 \\
\hline
\end{tabular}

Values are means \pm SEM.

\section{Discussion}

Vangl2 is a core component of PCP proteins, which are involved in cell-signaling that acts to divide and move cells along the tissue axis. Vangl2 comprises four transmembrane regions with both terminals exposed to the cytosol (Fig. 1A) (Tissir \& Goffinet, 2013; Yoshioka et al., 2013). In a previous report, we showed that Vangl2 is involved in bidirectional regulation of neuronal dendritic branching; a Vangl2N deletion mutant showed a significant reduction in dendritic branching and spine density (Hagiwara et al., 2014). Therefore, in this report, we searched for molecules that interact with Vangl2N to better understand the molecular mechanism underlying neural dendritic development. From the $\mathrm{Y} 2 \mathrm{H}$ screening, we obtained 61 candidate genes including pre- and post-synaptic proteins (Table 1) and confirmed the Vangl2N specific binding to adaptor protein Ap2m1(Fig. 1, 2, 3). Furthermore, shRNA KD of Ap2m1 and KD of Vangl2N resulted in less dendritic branching, although spine density increased after Ap2m1 KD (Fig. 4). From these results, we suggest that dendritic development associated with the clathrin-mediated endocytosis could be directed by the PCP pathway.

The C-terminal region of Vangl2 has multiple domains, including the looptail mutations D255E, S464N, and R259L, which cause neural tube defects, as well as interaction sites with numerous proteins, including Prickle (Pk) and PSD-95 (Yoshioka et al., 2013; Nagaoka et al., 2015; Bailly et al., 2018). Binding 
of Pk2 to the PCP protein binding region (Prickle binding domain; PKBD) of Vangl2 is required for dendritic spine formation (Nagaoka, Ohashi, et al., 2014). Considering synaptic proteins, Vangl2 forms a complex with PSD-95 through a conserved PDZ binding motif, which is required for localization of Vangl2 to the dendritic spines (Yoshioka et al., 2013). Thus, interacting molecules and functional roles of Vangl2 $\mathrm{C}$-terminus have been investigated in terms of neuronal morphology. However, even though the $\mathrm{N}$ terminus has an essential role in neuronal dendritic development, interaction at the $\mathrm{N}$-terminal region has not been well demonstrated (Hagiwara et al., 2014).

In mice, cortical layer 2/3 neurons have immature dendrites at $\mathrm{P} 3$, and apical and basal dendrites become longer at P5 and more complex by P15 (Hoshiba et al., 2016). Because Vangl2 expression gradually decreases during this dendritic morphogenesis during the first 1-2 weeks (Yoshioka et al., 2013), we screened molecules interacting with the $\mathrm{N}$-terminal region of Vangl2 using P9 mouse brain CDNA, and then identified Ap2m1 as a binding partner. In the pull-down assay using GST fused Vangl2 and Ap2m1expressing HEK293T lysates, Ap2m1 strongly bound to Vangl2N and weakly bound to Vangl2C (Fig. 1C and 3B). Consistent with these data, in the pull-down assay using GST fused Ap2m1 and Vangl2expressing HEK293T lysates, Ap2m1 bound to not only Vangl2 $\Delta \mathrm{C}$ but also Vangl2 $\Delta \mathrm{N}$ (Fig. 2B). These results suggested $A p 2 m 1$ preferentially bound to the $\mathrm{N}$-terminus of Vangl2, although it bound to both the $\mathrm{N}$ - and C-terminal regions. A previous study showed that Ap1m1, an AP-1 subunit, binds to the C-terminal YYXXF motif of Vangl2, which is required for Vangl2 transport from the trans-Golgi network with the GTPbinding protein, Arfrp1 (Guo et al., 2013). In the present study, we showed that compared with GSTVangl2-4 (corresponding to PKBD), Ap2m1 preferably interacted with the Vangl2 C-terminal, and hardly interacted with GST-Vangl2-3, which contains a YYXXF motif (279-283aa) (Fig. 3B). Therefore, the functional role of the interaction between AP-2 and Vangl2C might differ from that between AP-1 and Vangl2C.

Furthermore, in hippocampal neurons, binding of Vangl2 to N-cadherin through PKBD enhances its internalization in a Rab5-dependent manner (Nagaoka, Ohashi, et al., 2014), while in epithelial cells binding to E-cadherin is similar (Nagaoka, Inutsuka, et al., 2014). Because several endocytic adaptors including AP-2, Dab-2, and Numb associate with E-cadherin for the internalization of clathrin-mediated endocytosis (Bruser \& Bogdan, 2017), AP-2 may play a role in Vangl2-related N-cadherin internalization by binding to Vangl2 and/or $\mathrm{N}$-cadherin.

Because disruption of PCP genes, including Vangl2, Dishevelled1, and Dapper-antagonist of catenin-1 in mice, cause reduced dendritic arborization in neurons (Rosso et al., 2005; Okerlund et al., 2010, 2016; Hagiwara et al., 2014), the Wnt/PCP pathway is essential for the formation of the dendrite, dendritic spine, and excitatory synapse. In this study, we showed that Ap2m1 KD also decreased dendritic length, the total number of tips, and the branch number in cortical neurons and the Vangl2 KD (Fig. 4). Clathrinmediated endocytosis (CME) regulated dendritic growth by mediating the internalization of receptors. Indeed, KD of the clathrin assembly protein CALM reduced dendrite length and the overall complexity of the neurites in cultured hippocampal neurons (Bushlin et al., 2008). KD of AP2b1, a subunit of the AP-2 complex, lowered the total number of dendritic tips in rat hippocampal neurons (Koscielny et al., 2018). 
Consistent with these results, the direct interaction between Vangl2 and Ap2m1 suggests that the Wnt/PCP pathway and CME functionally coordinate the development of dendritic morphology.

\section{Materials And Methods}

The use of animals was approved by the Institutional Committee for the Care and Use of Experimental Animals at the University of Yamanashi (protocol \#A25-33, \#A30-21). All experiments were conducted according to the recommendations in the Guidelines for Proper Conduct of Animal Experiments of the Science Council of Japan (2006). And this study was carried out in compliance with the AARIVE (Abunak Research: Reporting of In Vivo experiments) guidelines.

\section{Yeast two-hybrid screening}

We screened a cDNA library from P9 ICR-mouse forebrain for Vangl2-interacting proteins using the Matchmaker Gold Yeast Two-Hybrid System (Clontech) according to the manufacturer's instructions. Briefly, random-primed cDNA was synthesized using poly $A^{+}$RNA taken from the P9 ICR-mouse forebrain using the SMART system (Clontech). The CDNA and PGADT7-Rec vector were co-transformed into the Y187 yeast strain (Clontech), which was plated on SD-lacking leucine (SD/-Leu) plates. Transformants were used for each screening. The Vangl2 amino acid residues 1-114 and 252-521 were subcloned into a pGBKT7 DNA-BD vector to yield pGBKT7-Vangl2 (1-114) and pGBKT7-Vangl2 (252-521), which were transformed into a $\mathrm{Y} 2 \mathrm{H}$ Gold yeast strain that was plated on SD/-Typ plates. The positive clone was then used to mate with the Y187 transformants and selected on SD/-Leu/-Trp plates supplemented with X-aGal and aureobasidin A (SD/-Leu/-Trp/X/A). The positive clones were subsequently selected on SD/-Leu/Trp/-Ade/-His/X/A plates. The yeast plasmid was transformed into E. coli and isolated DNA was sequenced.

\section{Construction of expression vectors}

The entire coding sequences of mouse, Ap2m1, Atp1b1, Eef1a1, Kif1a, and SynGAP1 were cloned into a pCAll-FLAG vector. The DNA fragment encoding amino acid residues 195-367 of Ap2m1 was amplified by PCR from the pCAII-FLAG-Ap2m1 vector and cloned into pGEX-4T-1 (GE Healthcare) and pCAll-EGFP vectors. The EcoRI-Sall fragment encoding amino acid residues 1-114 of Vangl2, and PCR amplified DNA fragments encoding residues 1-65, 66-114, 240-297, 298-382, 383-452, and 453-521 from Vangl2-were cloned into a pGEX-4T-1 vector.

\section{Pull-down assay}

GST, GST-VangI2N, GST-Vangl2C ${ }^{480-521}$ (Yoshioka et al., 2013), GST-Vangl2-1, -2, -3, -4, -5, -6, and GSTAp2m1 195-367) was expressed in E. Coli and lysed by sonication on ice. The bacteria lysate was used as a bait to pull down an interactor from the lysate of HEK293T cells that expressed a protein of interest. The lysates of bacteria and HEK293T cells were mixed and incubated for $2 \mathrm{~h}$ at $4^{\circ} \mathrm{C}$, followed by incubation with Glutathione Sepharose $4 \mathrm{~B}$ beads (GE Healthcare) overnight at $4^{\circ} \mathrm{C}$. After the beads were 
extensively washed with lysis buffer (20 mM Tris-Cl, pH 7.5, $150 \mathrm{mM} \mathrm{NaCl}, 0.5 \mathrm{mM}$ EDTA, 1 mM DTT, 1\% $[\mathrm{w} / \mathrm{v}$ ] Triton X-100, protease inhibitors [Complete EDTA-free, Roche]), the bound proteins were eluted from the beads by boiling in SDS sample buffer and analyzed by western blotting.

\section{Immunohistochemistry}

HEK293 cells were transfected with either or both of HA-Vangl2 and EGFP-Ap2m1 for 48 hours. Cells were fixed with $4 \%$ paraformaldehyde (PFA) and immunostained with the anti-HA antibody. Fluorescent images were taken with confocal microscopy (FV1200, Olympus)

\section{Immunoprecipitation}

Expression vectors pCAll-HA-Vangl2 and pCAII-FLAG-Ap2m1 were transfected into HEK293T cells. After 2 days, transfected cells were lysed with radioimmunoprecipitation (RIPA) buffer (50 mM Tris-Cl, pH 8.0, $150 \mathrm{mM} \mathrm{NaCl}, 0.1 \%$ SDS, $0.5 \%$ sodium deoxycholate, $1 \%$ Nonident P-40, protease inhibitors). Soluble fractions were incubated with anti-FLAG for $2 \mathrm{~h}$ at $4^{\circ} \mathrm{C}$, followed by incubation with Protein $\mathrm{G}$ Sepharose beads (GE Healthcare) overnight at $4^{\circ} \mathrm{C}$. After the beads were extensively washed with RIPA buffer, the bound proteins were eluted from the beads by boiling in SDS sample buffer and analyzed by western blotting.

\section{ShRNA}

Oligonucleotides were annealed and ligated into BgllI/Xhol site of pSUPER.neo+gfp vector (Oligoengine) to yield pSUPER-Ap2m1 vector according to the manufacturer's instructions. The target sequence of shRNA against Ap2m1 was 5'-CAAAGGCACAGCTGATGAAAC-3'. The knockdown efficiency of shRNA was determined in HEK293T cells by cotransfection with pCAll-FLAG-Ap2m1. Three days after transfection, transfected cells were extracted with SDS sample buffer and analyzed by western blotting. The intensities of bands for Ap2m1 were quantified and normalized with those for GFP. The scrambled non-effective shRNA (5'-GAAACGGAAAGCAGGTACG-3') (Hagiwara et al., 2014) was used as a control.

\section{In utero electroporation}

All experiments were performed in accordance with relevant guidelines including AARIVE. In utero electroporation was performed as described previously (Saito \& Nakatsuji, 2001; Tabata \& Nakajima, 2001). Briefly, pregnant ICR mice at embryonic day (E) 14.5 or E15.5 were anesthetized with $10 \%$ pentobarbital solution $(0.1 \mathrm{~mL} / 10 \mathrm{~g}$ body weight), and the uterine horns were exposed. Approximately 1-2 $\mathrm{ml}$ of DNA solutions $(0.5 \mathrm{mg} / \mathrm{ml}$ pCAll-EGFP with $1 \mathrm{mg} / \mathrm{ml}$ pSUPER-Scramble, pSUPER-Vangl2 (Hagiwara et al., 2014), or pSUPER-Ap2m1) containing $0.01 \%$ fast green was injected into the lateral ventricles of embryos using pulled borosilicate glass capillaries (B120F-4; World Precision Instruments). The head of an embryo in the uterus was pinched with a forceps-type electrode (CUY650P5; NEPA Gene) and five square electric pulses (33 V, $50 \mathrm{~ms}$ ) at intervals of $950 \mathrm{~ms}$ were delivered using an electroporator (CUY21E; NEPA Gene). After electroporation, the embryos were returned to the abdominal cavity to allow continuous development. 


\section{Histological analysis}

Under deep anesthesia, mice were fixed transcardially with 4\% PFA in PBS (pH 7.4) at P21. Coronal sections $(50 \mathrm{~mm})$ were embedded and images were acquired with confocal laser microscopy (FV-1200, Olympus). Apical and basal dendrites of the cortical layer 2/3 pyramidal neurons were randomly sampled and analyzed using Amira 5.5 software (FEI).

\section{Statistical methods}

Statistical significance was evaluated by Student's t-test or one-way ANOVA followed by post hoc Tukey's test. Statistical significance was assumed when $p<0.05$.

\section{Declarations}

\section{Acknowledgments}

This work was supported by JSPS KAKENHI Grant Numbers 17K14949 (to Y.H.) and 19H03324 (to T.O.) and the University of Yamanashi. We thank T. Okazaki, T. Maeda, and all members of the Ohtsuka Laboratory for their helpful discussions and technical assistance. The experiment of in utero electroporation was supported by Dr. T. Mori at Shishu University, and Dr. J. Hatakeyama and Dr. K. Shimamura at the Joint Usage/Research Center for Developmental Medicine, IMEG, Kumamoto University. We also wish to thank Adam Phillips, PhD, from Edanz Group (https://en-authorservices.edanz.com/ac) for editing a draft of this manuscript.

\section{Author Contributions}

MY designed and performed the biochemical, and anatomical analysis with the support of $\mathrm{AH}$ and $\mathrm{YH}$, and $\mathrm{MY}$ and $\mathrm{AH}$ drafted the manuscript. TO directed the study and edited the manuscript.

\section{Competing Interests}

The authors declare that the research was conducted in the absence of any commercial or financial relationships that could be construed as a potential conflict of interest.

\section{Data Accessibility}

The datasets used and/or analyzed during the current study are available from the corresponding author on reasonable request.

\section{References}

1. Bailly, E., Walton, A. \& Borg, J. The planar cell polarity Vangl2 protein: From genetics to cellular and molecular functions. Semin. Cell Dev. Biol. 81, 62-70 (2018). 
2. Bruser, L. \& Bogdan, S. Adherens junctions on the move - Membrane trafficking of E-cadherin. Cold Spring Harb. Perspect. Biol. 9, a029140 (2017).

3. Bushlin, I. et al. Clathrin assembly protein AP180 and CALM differentially control axogenesis and dendrite outgrowth in embryonic hippocampal neurons. J Neurosci. 28, 10257-10271 (2008).

4. Guo, Y., Zanetti, G. \& Schekman, R. A novel GTP-binding protein-adaptor protein complex responsible for export of Vangl2 from the trans Golgi network. Elife. 2, e00160 (2013).

5. Hagiwara, A., Yasumura, M., Hida, Y., Inoue, E. \& Ohtsuka, T. The planar cell polarity protein Vangl2 bidirectionally regulates dendritic branching in cultured hippocampal neurons. Mol. Brain. 7, 1-6 (2014).

6. Hoshiba, Y. et al. Sox11 balances dendritic morphogenesis with neuronal migration in the developing cerebral cortex. J Neurosci. 36, 5775-5784 (2016).

7. Koscielny, A. et al. Adaptor complex 2 controls dendrite morphology via mTOR-dependent expression of GluA2. Mol Neurobiol. 55, 1590-1606 (2018).

8. Nagaoka, T., Inutsuka, A., Begum, K., Bin hafiz, K. M. \& Kishi, M. (2014) Vangl2 regulates E-cadherin in epithelial cells.Sci. Rep., 4.

9. Nagaoka, T. \& Kishi, M. The planar cell polarity protein Vangl2 is involved in postsynaptic compartmentalization. Neurosci. Lett. 612, 251-255 (2016).

10. Nagaoka, T. et al. The Wnt/planar cell polarity pathway component Vangl2 induces synapse formation through direct control of N-cadherin. Cell Rep. 6, 916-927 (2014).

11. Nagaoka, T., Tabuchi, K. \& Kishi, M. (2015) PDZ interaction of Vangl2 links PSD-95 and Prickle2 but plays only a limited role in the synaptic localisation of Vangl2.Sci. Rep.,1-9.

12. Okerlund, N. D. et al. Dact1 is a postsynaptic protein required for dendrite, spine, and excitatory synapse development in the mouse forebrain. J Neurosci. 30, 4362-4368 (2010).

13. Okerlund, N. D., Stanley, R. E. \& Cheyette, B. N. R. The planar cell polarity transmembrane protein Vangl2 promotes dendrite, spine and glutamatergic synapse formation in the mammalian forebrain. Mol. Neurophyciatry. 2, 107-114 (2016).

14. Rosso, S. B., Sussman, D., Wynshaw-Boris, A. \& Salinas, P. C. Wnt signaling through Dishevelled, Rac and JNK regulates dendritic development. Nat. Neurosci. 8, 34-42 (2005).

15. Saito, T. \& Nakatsuji, N. Efficient gene transfer into the embryonic mouse brain using in vivo electroporation. Dev. Biol. 240, 237-246 (2001).

16. Shafer, B., Onishi, K., Lo, C., Colakoglu, G. \& Zou, Y. Vangl2 promotes Wnt/Planar cell polarity-like signaling by antagonizing Dvl1-mediated feedback inhibition in growth cone guidance. Dev. Cell. 20, 177-191 (2011).

17. Shao, G. et al. Proteomic analysis of mouse cortex postsynaptic density following neonatal brain hypoxia-ischemia. Dev Neurosci. 39, 66-81 (2017).

18. Tabata, H. \& Nakajima, K. Efficient in utero gene transfer system to the developing mouse brain using electroporation: visualization of neuronal migration in the developing cortex. Neuroscience. 103, 
865-872 (2001).

19. Tissir, F. \& Goffinet, A. M. Expression of planar cell polarity genes during development of the mouse CNS. Eur J Neurosci. 23, 597-607 (2006).

20. Tissir, F. \& Goffinet, A. M. Shaping the nervous system: role of the core planar cell polarity genes. Nat. Rev. Neurosci. 14, 525-535 (2013).

21. Torban, E., Kor, C. \& Gros, P. Van Gogh-like2 (Strabismus) and its role in planar cell polarity and convergent extension in vertebrates. Trends Genet. 20, 570-577 (2004).

22. Yoshioka, T., Hagiwara, A., Hida, Y. \& Ohtsuka, T. Vangl2, the planner cell polarity protein, is complexed with postsynaptic density protein PSD-95. FEBS Lett. 587, 1453-1459 (2013).

Figures

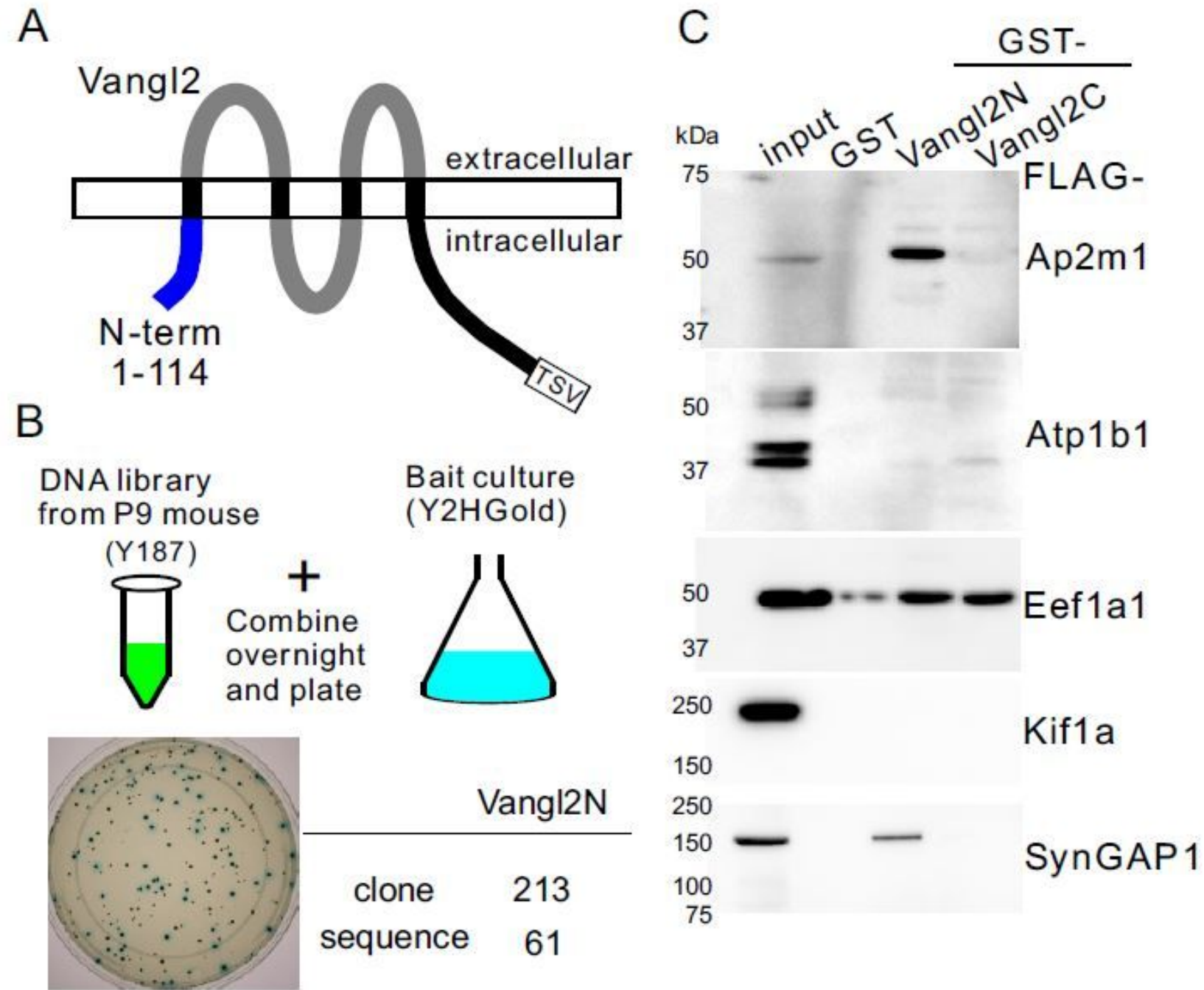

Figure 1 
Screening of Vangl2 interacting partners using yeast two-hybrid. (A) Structure of the Vangl2 protein includes 4 transmembrane regions (black boxes, 111-126, 148-166, 183-202, 218-237), and a PDZ binding motif (TSV, 519-521). To detect novel Vangl2 interacting-partners, the $\mathrm{N}$-terminal (1-114) region of Vangl2 was incorporated as the bait plasmid. (B) The bait culture of Vangl2N was combined with prey plasmids of the P9 mouse DNA library and then plated. The positive clones were identified via blue color staining. (C) To confirm the screening results of the yeast two-hybrid test, binding of Vangl2N and Cterminal (242-521) proteins were investigated by pull-down assay via western blotting using anti-FLAG antibody. From the five post-synaptically localized proteins, Ap2m1 and synGAP1 bound strongly to the $\mathrm{N}$-terminal region rather than the $\mathrm{C}$-terminal region of Vangl2. The full-length western blots were presented in Supplementary Fig.1.

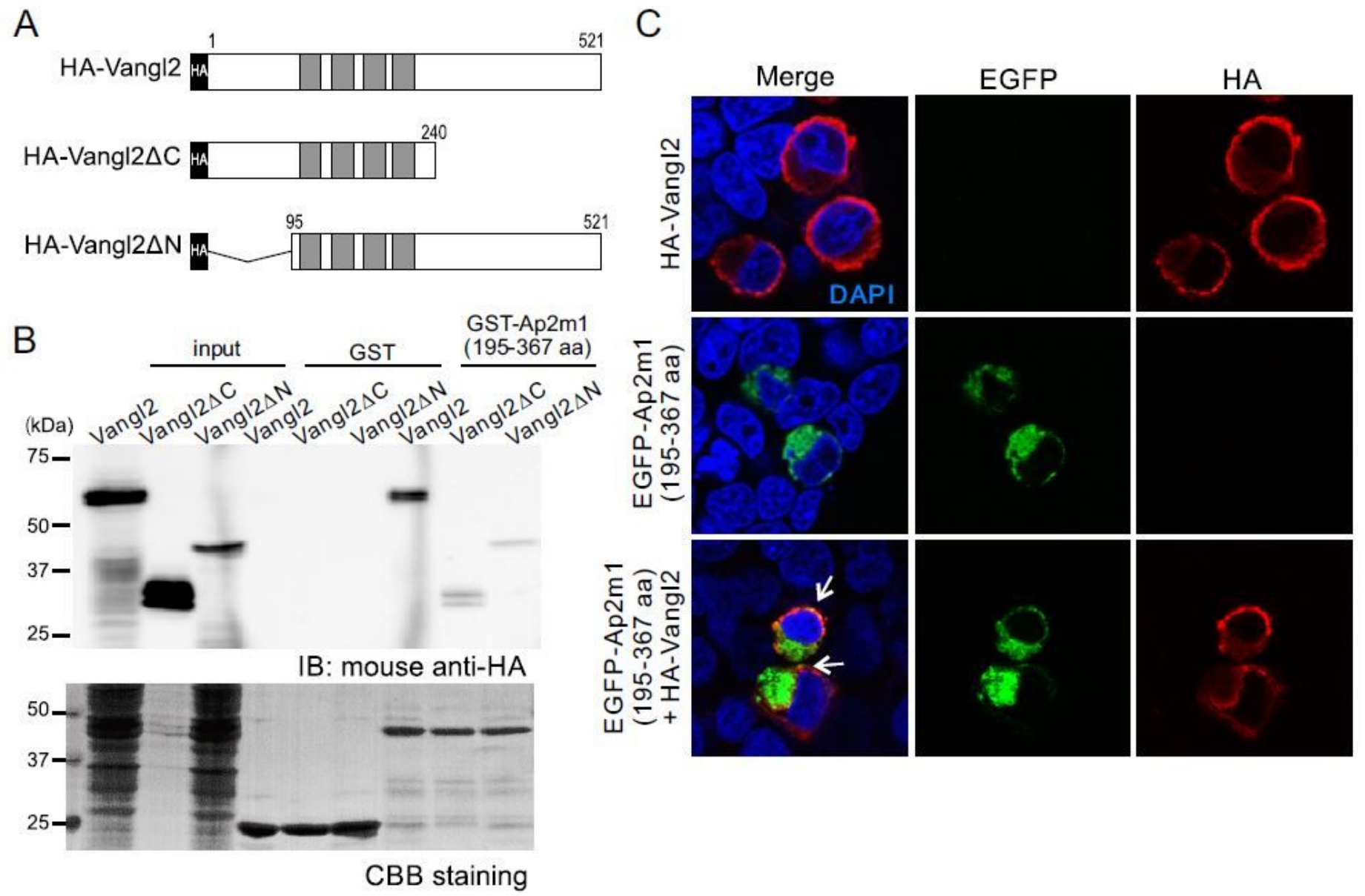

\section{Figure 2}

Binding of Vangl2 with Ap2m1 (A, B) The binding of Vangl2 with Ap2m1 was confirmed by a pull-down assay using Vangl2 deletion mutants. The HA-tagged Vangl2, C-terminal deletion mutant (Vangl $2 \Delta C)$, and N-terminal deletion mutant (Vangl $2 \Delta \mathrm{N})(\mathrm{A})$ were incubated with GST-fusion of Ap2m1 and detected with anti-HA antibody by western blotting (B). Ap2m1 robustly interacted with the full length of Vangl2. However, binding of Ap2m1 was reduced by the deletion of the $\mathrm{N}$-terminal and the C-terminal deletion. The full-length western blots were presented in Supplementary Fig.1. (C) Colocalization of Vangl2 and 
Ap2m1 by confocal microscopy. HA and EGFP labeled Vangl2 and Ap2m1 were transfected in HEK293 cells and the expression was observed via confocal microscopy. Co-expression of HA-Vangl2 and EGFPAp2m1 revealed the colocalization of these proteins (arrows).

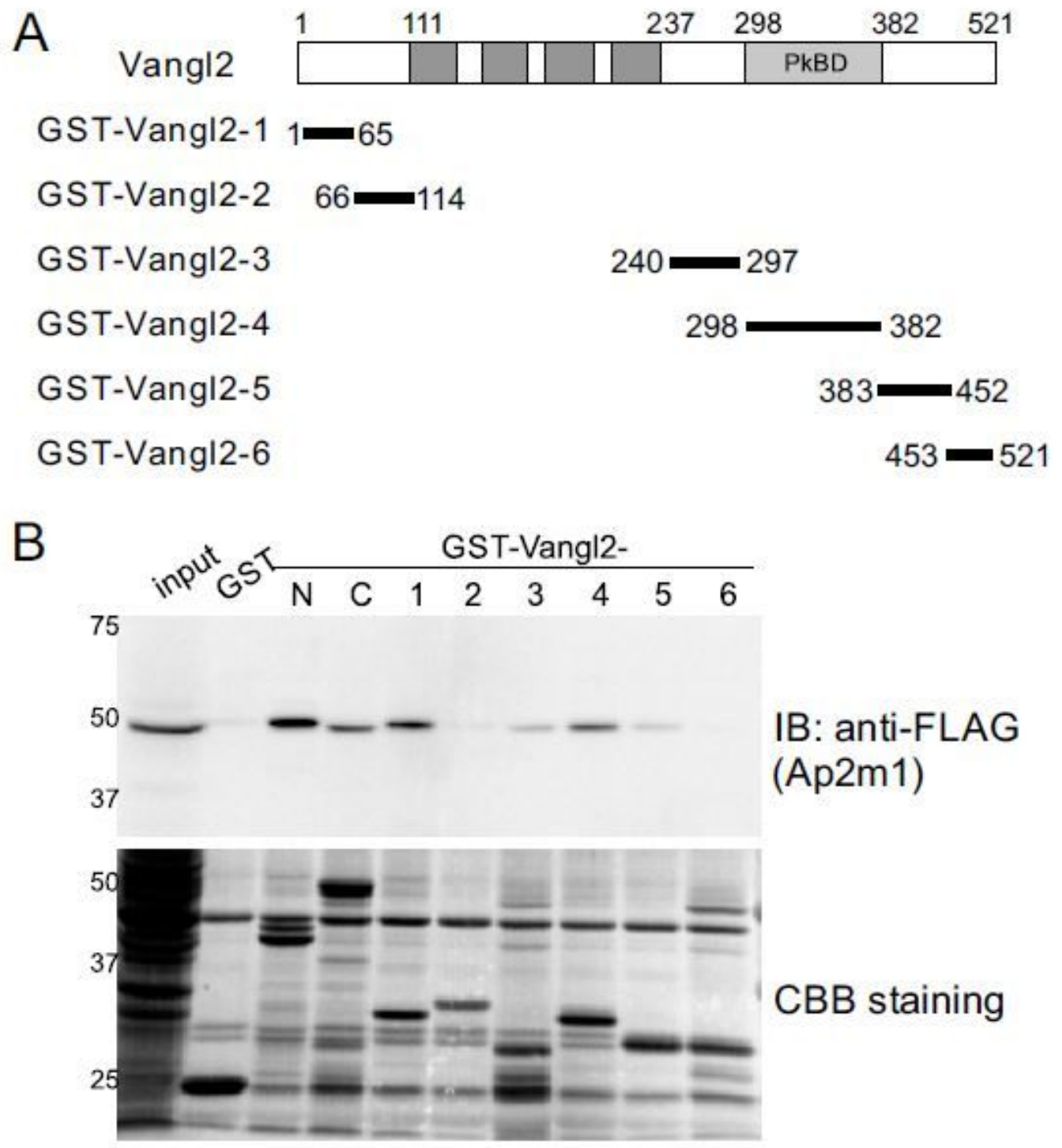

\section{Figure 3}

Confirmation of the Vangl2-Ap2m1 binding region (A) Constructs of Vangl2 for GST fusion proteins. The constructs excluded the transmembrane regions (gray boxes from 111 to 237). (B) Pull-down assay for the Ap2m1 domain of Vangl2. Ap2m1 strongly interacted with the $\mathrm{N}$-terminal $(\mathrm{N})$ and the $\mathrm{C}$-terminal (C). The band could be detected for Vangl2-1 (1-65) and Vangl2-3, 4, and 5 (240-452), including the Prickle binding domain (PkBD). The PDZ binding motif in Vangl2-6 (453-521) was less essential for the binding of Ap2m1. The full-length western blots were presented in Supplementary Fig.1. 


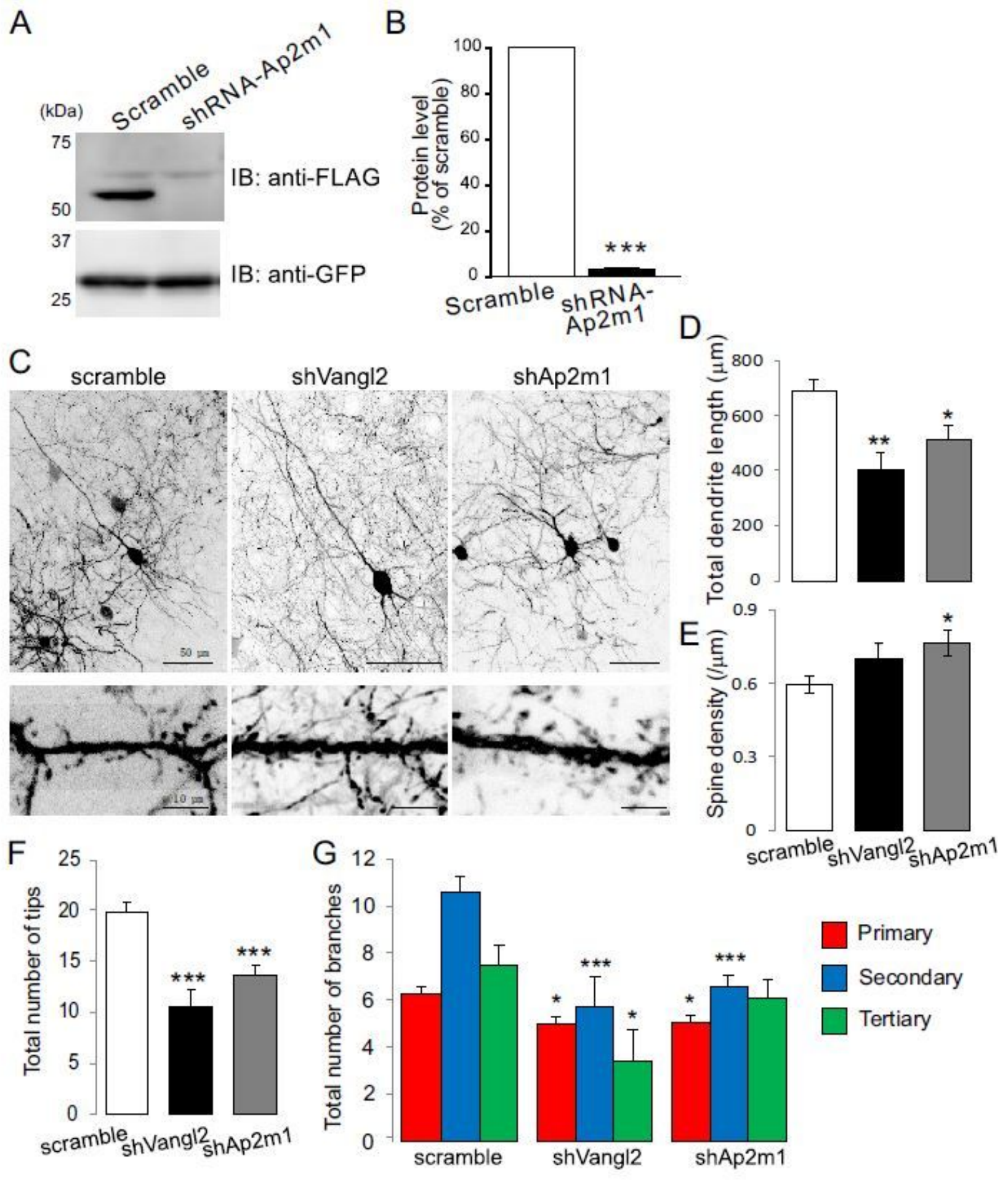

\section{Figure 4}

Knockdown of Ap2m1 and Vangl2 affects neuronal dendritic branching (A, B) The knockdown of shRNAAp2m1 on transiently expressed FLAG-Ap2m1 was evaluated with western blotting. From three independent tests, the protein level was suppressed about $3.0 \% \pm 0.9 \%$. Data are means $\pm S E M, * \star \star p<$ 0.001, Student's t-test. The full-length western blots were presented in Supplementary Fig.1. (C) Morphology of cortical neurons as revealed by co-expressed EGFP with knockdown vectors. Spine and tip number were measured with high magnification of apical and basal dendrites (bottom). (D-G) The 
dendritic and spine complexity of neurons expressing the indicated shRNA constructs. Dendritic expansion as measured by total dendrite length was significantly reduced in Vangl2 and Ap2m1 KD neurons, and the spine density was increased in Ap2m1 KD. The number of tips was significantly less in Vangl2 and Ap2m1 KD. Specifically, the numbers of branches on the secondary dendrites were remarkably less in Vangl2 and Ap2m1 KD. Data are means \pm SEM, ${ }^{\star} p<0.05, * \star p<0.01 * \star * p<0.001$, one-way ANOVA post hoc Tukey test.

\section{Supplementary Files}

This is a list of supplementary files associated with this preprint. Click to download.

- Scientificreportssupple210113.docx 